\title{
A chance diagnosis of Pelger-Huët anomaly in a 49-year-old woman hospitalized for an acute episode of Crohn disease
}

\section{Diagnostic fortuit d'une anomalie de Pelger-Huët chez une patiente de 49 ans hospitalisée pour une poussée aiguë de maladie de Crohn}

\author{
Anaïs Schavgoulidze \\ Francois Vergez \\ Jill Corre \\ Jean-Baptiste Rieu \\ Haematology laboratory, Cancer \\ university institute of \\ Toulouse-Oncopole, Toulouse, France
}

Article received May 05, 2020,

accepted June 16, 2020

A 49-year-old woman was admitted with an acute episode of Crohn disease. She had stopped taking her treatment (prednisone + azathioprine) several months ago. She had neither other treatments nor relevant medical history. Clinical examination was otherwise normal as were the results of the blood count. In the blood film, all the polymorphonuclear leukocytes without exception have a hyposegmented nucleus and abnormally condensed chromatin (May-Grünwald-Giemsa, objective $\times 100$ ). Three shapes of nucleus can be distinguished: a single round nucleus (figure 1A), an elongated nucleus in the shape of a bean or a peanut (figure $1 B$ ), and a bilobed nucleus with a "pince-nez" or "dumbbell" shape (figure 1C). All three are characteristic of Pelger-Huët anomaly (PHA).

Heterozygous mutations of LBR gene, coding for the lamin $\mathrm{B}$ receptor (LBR), are responsible for PHA. PHA is entirely asymptomatic. The cytological anomaly involves all polymorphonuclear leukocytes (neutrophils, eosinophils and basophils) but does not affect their physiological functions.
PHA is not detected by haematology analysers. It is often a chance diagnosis and raises the problem of the differential diagnosis with most common acquired Pelger-Huët anomaly (APHA). Investigation of family members is recommended. Cytological examination of the blood film is sufficient to identify individuals who are carriers of the mutation. Search for LBR mutations by molecular genetic analysis is not routinely done.

Homozygous mutations are exceptional. They may be responsible for severe abnormalities, often including skeletal dysplasia, whose most serious form (Greenberg dysplasia) is fatal in utero. These manifestations are probably due to defective sterol reductase activity of the LBR (in heterozygous individuals, the decreased number of functional LBRs has no metabolic consequences) [1]. In view of the risk of severe presentations in homozygous individuals and composite heterozygous individuals, genetic advice is nonetheless necessary. 


\section{Biological pictures}

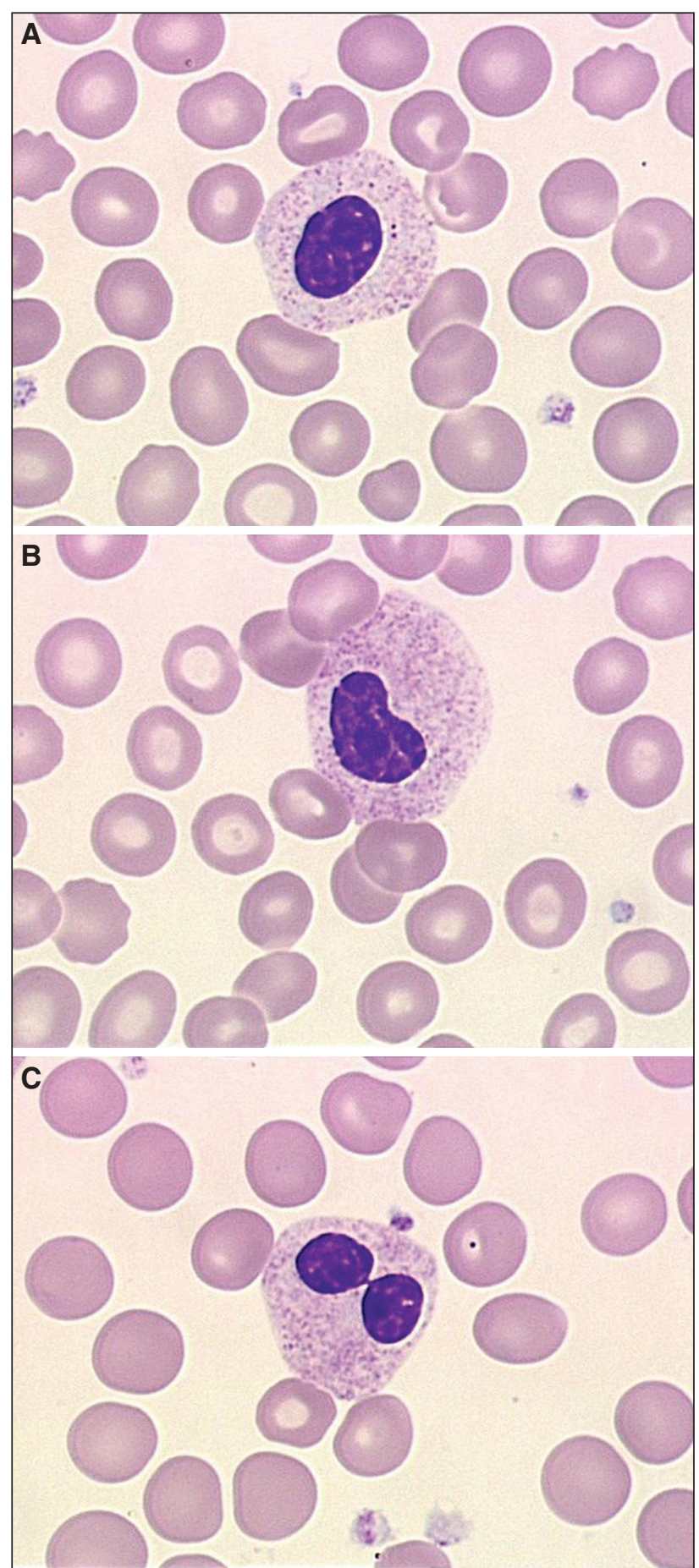

Figure 1. Three characteristic shapes of nucleus in Pelger-Huët anomaly: A- single round nucleus, B- elongated nucleus in the shape of a bean or a peanut, C- bilobed nucleus with a "pince-nez" or "dumbbell" shape (blood smear, May-Grünwald-Giemsa staining, objective $\times 100$ ).
Conflict of interest: none of the authors has any conflict of interest to disclose concerning this article.

\section{Reference}

1. Hoffmann K, Dreger CK, Olins AL, Olins DE, Shultz DL, Lucke B, et al. Mutations in the gene encoding the lamin B receptor produce an altered nuclear morphology in granulocytes (Pelger-Huët anomaly). Nat Genet $2012 ; 31(4): 410-4$. 